\title{
PROSPETTIVE DI RICERCA SULL'ETEROGLOSSIA A BASE ITALIANA
}

\author{
GIOVANNI IAMARTINO (*)
}

SuNTO. - Prendendo spunto dalle relazioni incluse nel volume e facendo anche riferimento alla letteratura critica esistente, il contributo si propone di trattare alcuni aspetti rilevanti circa la natura dell'eteroglossia letteraria e di suggerire ulteriori riflessioni e prospettive di ricerca.

$$
* * *
$$

ABSTRACT. - By referring to the contributions included in this volume and pertinent critical literature, this paper aims to deal with some relevant features of literary heteroglossia and to suggest further reflections and research perspectives.

1. Gli interventi sull'eteroglossia a base italiana che hanno dato vita alla giornata di studio presso l'Istituto Lombardo Accademia di Scienze e Lettere e che ora sono raccolti in questo volume costituiscono degli interessanti studi di caso per un duplice motivo: da una parte, in quanto si concentrano sul Sette-Ottocento, probabilmente il periodo più trascurato dagli studiosi dell'eteroglossia rispetto a epoche più diffusamente caratterizzate da plurilinguismo ed eteroglossia come il Cinque-Seicento e il Novecento; ${ }^{1}$ dall'altra, in quanto pongono, esplici-

${ }^{(*)}$ Istituto Lombardo Accademia di Scienze e Lettere; Università degli Studi di Milano, Italia. E-mail: giovanni.iamartino@unimi.it

1 Con riferimento alla prima età moderna, sono ancora imprescindibili Ivano Paccagnella, Il fasto delle lingue. Plurilinguismo letterario nel Cinquecento, Roma, Bulzoni, 1984 e Gianfranco Folena, L'italiano in Europa. Esperienze linguistiche del Settecento, Torino, Einaudi, 1983. Per una più ampia veduta d'insieme e interessanti studi di caso si deve fare riferimento ai lavori scritti e/o curati da Furio Brugnolo: Furio Brugnolo e Vincenzo Orioles (a cura di), Eteroglossia e plurilinguismo letterario (vol. 1: L'italiano in Europa; vol. 2: Plurilinguismo e letteratura), Roma, Il Calamo, 2002; Furio Brugnolo, La lingua di cui si vanta Amore. Scrittori stranieri in lingua italiana dal 
tamente o meno, significative questioni di carattere metodologico. In sintesi, si è voluto capire e mostrare come si gioca il rapporto fra la norma linguistica di un determinato modello d'italiano e l'uso che ne fa - più o meno adeguato alla norma, più o meno ostacolato dalla propria lingua madre, più o meno rispondente ai propri bisogni comunicativi un letterato o uomo di cultura straniero. ${ }^{2}$

Gli interventi qui raccolti in volume, tuttavia, hanno anche sollevato e discusso altri problemi legati alla natura e all'uso della scrittura in lingua straniera. Merita quindi trarre da tali interventi, e dalla bibliografia critica disponibile sull'eteroglossia e su tematiche a essa correlate, alcune considerazioni che possano fornire stimoli per ulteriori ricerche. Tali considerazioni verranno qui di seguito presentate, per comodità espositiva, distinguendo le prospettive di ricerca sull'eteroglossia di taglio linguistico, letterario, e culturale in senso lato.

2. Focalizzando l'attenzione sull'eteroglossia dal punto di vista degli studi linguistici, è innanzitutto utile ricordare che, mentre l'indagine linguistica - di taglio sincronico oppure diacronico - si occupa solitamente del codice linguistico, della sua natura e dei suoi mutamenti, lo studio dell'eteroglossia si focalizza su uno specifico testo che dà attuazione a quel codice; detto altrimenti, i documenti linguistici dell'eteroglossia letteraria non possono che rappresentare un idioletto, l'uso

Medioevo al Rinascimento, Roma, Carocci, 2009; Furio Brugnolo (a cura di), Scrittori stranieri in lingua italiana dal Cinquecento ad oggi, Padova, Unipress, 2009. Naturalmente, questa bibliografia essenziale può essere integrata dai riferimenti contenuti nei saggi che compongono questo volume.

2 Il particolare interesse per la norma linguistica è stato anche determinato dal fatto che la giornata di studio presso l'Istituto Lombardo Accademia di Scienze e Lettere - di cui questo volume è testimonianza - rappresentava il momento ultimo del progetto di ricerca PRIN 2009WFSAAK, da me coordinato e intitolato "Entro e oltre i confini: uso e norma nelle lingue dell'Europa occidentale". Tale progetto aveva l'obiettivo di analizzare il rapporto tra la norma linguistica - codificata in dizionari, grammatiche e manuali - e il concreto uso dei parlanti, senza dimenticare l'atteggiamento dei parlanti stessi nei confronti della norma, a volte osteggiata o trascurata, altre volte auspicata e seguita. il riferimento ai 'confini' nel titolo del progetto serviva a porre la questione della dialettica norma/uso nella prospettiva del plurilinguismo, da intendere in vari modi: in riferimento a realtà linguistiche e sociopolitiche diverse, al plurilinguismo insito in ciascuna comunità linguistica, e al plurilinguismo individuale di singoli parlanti, requisito essenziale, questo, dell'eteroglossia. 
linguistico specifico di un determinato scrivente o scrittore; un uso, poi, del tutto particolare, visto che si esercita attraverso una lingua straniera e non la lingua madre - o forse, meglio, attraverso tale lingua altra in rapporto dialettico con la lingua madre.

In questa prospettiva, va ripreso il concetto di eteroglossia elaborato da Michail Bachtin e ricordare che tutti i parlanti sono plurilingui, tutti praticano l'eteroglossia anche usando la propria lingua madre: esistono infatti diverse varietà all'interno di ciascuna lingua - varietà legate all'età, al genere, al livello d'istruzione, all'appartenenza sociale e professionale, all'ambito d'uso etc. Sebbene tale concetto fosse stato originariamente elaborato da Bachtin nel contesto della sua riflessione sul romanzo e analizzando l'intento dei romanzieri di ricreare tali varietà all'interno delle proprie opere con finalità stilistiche e letterarie, il concetto e il relativo termine vennero presto estesi a definire la produzione di testi in una lingua diversa da quella materna. ${ }^{3}$

Su tali fondamenti, volendo stabilire a che cosa potrebbe mirare un'analisi linguistica del fenomeno dell'eteroglossia, si dovrebbe innanzitutto tentare di determinare il modello di lingua altra a cui si rifà lo scrittore: ${ }^{4}$ potrebbe un modello classico e tradizionale, oppure il comune uso contemporaneo oppure ancora una variante non standard e innovativa della lingua. Per fare un esempio, quando negli anni Venti dell'Ottocento, l'avvocato francese Boudet de Montesquieu si trasferisce a Firenze e si dedica alla compilazione di una grammatica, egli pratica l'uso fiorentino del tempo ma nella sua grammatica si riferisce al modello Tre-Quattrocentesco. ${ }^{5}$

Andrebbe anche determinato dove e come nasce la competenza

3 Il pensiero critico di Michail Bachtin è convenientemente raccolto in Pam Morris (a cura di), The Bachtin reader, Oxford, Oxford University Press, 1994.

4 Le presenti considerazioni si focalizzano sull'eteroglossia letteraria, ovvero la produzione di testi scritti in lingua straniera con finalità letteraria o paraletteraria. Ben diverso, per la natura e l'estensione del fenomeno, è il caso dell'eteroglossia a livello comunicativo (specialmente, della comunicazione orale): su questo si vedano, tra gli altri, Joan Pujolar, Gender, heteroglossia and power. A sociolinguistic study of youth culture, Berlino, De Gruyter, 2001, Adrian Blackledge e Angela Creese (a cura di), Heteroglossia as practice and pedagogy, Dordrecht, Springer, 2014, e alcuni dei saggi in Gabriella Cartago e Giovanni Rovere (a cura di), "Verso nuove frontiere dell'eteroglossia”, numero monografico di Lingue Culture Mediazioni, 3 (1), 2016.

5 Si veda il saggio di Anne Schoysman in questo volume. Anche più sotto, menzionando i nomi degli autori, faccio riferimento ad alcuni degli altri saggi qui raccolti. 
nella lingua straniera da parte di chi si dedica all'eteroglossia. Va chiarito infatti se si tratta di una competenza linguistica fondata su una pratica libresca o su una reale esperienza di vita all'estero o ancora su una commistione delle due. Sarebbe pure importante riscontrare a livello biografico se tale competenza sia frutto di un bilinguismo acquisito in giovane età o maturato in età adulta. In questa prospettiva, sarebbe interessante provare a determinare di quali supporti linguistici - dizionari mono e bilingui, grammatiche, manuali - si sia eventualmente avvalso l'autore di scritti in lingua straniera, e in quale misura la norma proposta o imposta da questi strumenti della codificazione linguistica abbia inciso sulla scrittura di chi produce un testo in lingua straniera.

Tutti questi elementi, che è talvolta possibile riscontrare a livello biografico o documentale (ad esempio, quando è reperibile un catalogo dei libri posseduti da un dato scrittore, o se esistono copie annotate di tali libri), si possono ritrovare, più o meno chiaramente, in alcuni tratti linguistici del testo in lingua straniera: un'adesione attenta e adeguata alla norma morfosintattica, o al contrario trascurata, può dire qualcosa sull'effettiva competenza linguistica dello scrittore; il peso dell'interferenza della lingua madre può essere correlato alla natura dell'interesse dello scrittore per l'eteroglossia; certe scelte lessicali e stilistiche possono far rimandare a determinati modelli letterari ovvero a una pratica d'uso colloquiale o strumentale della lingua straniera; e così via.

3. Molte delle considerazioni appena fatte portano senza soluzione di continuità dalla ricerca di taglio linguistico a quella di taglio letterario. In questa seconda prospettiva sembra essere di primaria importanza valutare le motivazioni - innanzitutto, quelle di carattere più ampio e generale - che possono essere sottese alla produzione di un testo in lingua straniera: da un lato, l'assunzione o l'imitazione di un modello letterario considerato prestigioso; dall'altro, l'inserimento del prodotto testuale dell'eteroglossia in una tradizione letteraria particolarmente apprezzata. Ovviamente, queste due motivazioni non si escludono a vicenda, specialmente se la lingua madre dell'autore e la tradizione letteraria in cui naturalmente questi si colloca godono di un inferiore prestigio a livello culturale sovranazionale.

Vanno poi considerate motivazioni di carattere più contingente, anche se non meno rilevanti in quanto correlate a scelte personali dell'autore coinvolto. Si può infatti individuare nella scelta dell'eteroglossia un 
intento puramente o primariamente ludico, il desiderio di fare della parodia verbale, giocare con le parole, con le loro sonorità e modalità creative, come ad esempio è stato almeno talvolta per l'italiano di James Joyce. ${ }^{6}$ Oppure la lingua straniera viene preferita alla propria in quanto - per l'autore così come per la sua comunità di appartenenza - è una lingua altra, dunque 'opaca', non trasparente, una lingua che si rende evidente prima e forse di più del messaggio che veicola. Oppure ancora tale decisione nasce nello scrittore dal desiderio di distacco, di oggettivazione del sé, come nel caso delle opere di Samuel Beckett in francese. ${ }^{7}$

Nel caso di un'indagine critica sull'eteroglossia a fine propriamente letterario, certamente lo scopo primo del ricercatore è cogliere nel testo la letterarietà, lo specifico letterario. Questo dato è particolarmente importante perché la scrittura in lingua straniera ha nel tempo trovato realizzazione in testi caratterizzati da diversi livelli di letterarietà; detto altrimenti, occorre distinguere fra gli usi più propriamente letterari e quelli paraletterari dell'eteroglossia. Infatti, il testo in lingua straniera può rientrare in un genere prototipico della tradizione letteraria, e quindi ad alto tasso di letterarietà, come ad esempio un sonetto. Diversamente, un resoconto di viaggio, un diario, una lettera, che in termini generali possono essere qualificati come testi paraletterari, vanno

6 Naturalmente, Joyce seppe andare ben al di là del giocare con le parole, visto che stese e pubblicò in italiano articoli giornalistici, testi di conferenze e saggi, oltre all'autotraduzione di brani della sua ultima opera, Anna Livia Plurabelle. Si vedano Gianfranco Corsini e Giorgio Melchiori (a cura di), James Joyce. Scritti italiani, Milano, Mondadori, 1979; Serenella Zanotti, "L'italiano di Joyce nell'autotraduzione di Anna Livia Plurabelle", in Furio Brugnolo e Vincenzo Orioles (a cura di), Eteroglossia e plurilinguismo letterario, vol. 2: Plurilinguismo e letteratura, Roma, Il Calamo, 2002, pp. 277-307; Massimo Bacigalupo, "La lingua di Finnegan: James Joyce saggista e poeta italiano", in Furio Brugnolo (a cura di), Scrittori stranieri in lingua italiana dal Cinquecento ad oggi, Padova, Unipress, 2009, pp. 361-373; Enrico Terrinoni, "La lettera che vive e il linguaggio alato", introduzione a James Joyce, Lettere e saggi, a cura di Enrico Terrinoni, Milano, Il Saggiatore, 2016, pp. 11-32. In una prospettiva più ampia è ancora utilissimo Giorgio Melchiori, Joyce. Il mestiere dello scrittore, Torino, Einaudi, 1994.

7 Si vedano tra gli altri: Brian T. Fitch, Beckett and Babel: An investigation into the status of the bilingual work, Toronto, University of Toronto Press, 1988; Michael Oustinoff, Bilinguism d'écriture et auto-traduction. Julien Green, Samuel Beckett, Vladimir Nabokov, Parigi, L'Harmattan, 2001; Rainier Grutman, "Beckett and beyond. Putting self-translation in perspective", Orbis Litterarum, 68 (3), 2013, pp.188-206; Rossana Sebellin, "Beckett tra scrittura e (auto)traduzione", Testo \& Senso, 19, 2018, pp. 1-17. 
attentamente valutati e distinti in relazione all'intento dell'autore e all'esito del suo lavoro: la letteratura di viaggio comprende testi già concepiti per la futura pubblicazione, e quindi redatti con piena cognizione di modelli e tradizioni, ma anche scritti in lingua straniera che nascono contingentemente dal contesto alloglotto in cui si viene a trovare il viaggiatore, il quale intende solo mettere alla prova la competenza linguistica che sta acquisendo o approfondendo. Allo stesso modo, diari e lettere possono essere stati scritti come 'documenti dell'io', in cui lo scrivente sceglie la lingua straniera per parlare di sé a sé stesso o a un interlocutore in un rapporto personale e privato, oppure con la chiara idea e prospettiva di una futura pubblicazione, con tutto ciò che questo implica in termini di scrittura.

Con riferimento particolare all'eteroglossia a base italiana, gli studi di caso raccolti in questo volume mostrano, sia la varietà e la gamma stilistico-letteraria a cui si è appena fatto cenno, sia la relativa predominanza della letteratura odeporica, indubbiamente favorita nel passato dall'apprendimento dell'italiano da parte di molti degli stranieri che si accingevano al Grand Tour. Inoltre, le ricerche qui proposte ci invitano a non stabilire correlazioni troppo rigide nel rapporto fra gli autori che praticano l'eteroglossia e la tipologia dei testi che producono: Francesco Rognoni dimostra ad esempio che un dato scrittore, nel suo caso Percy Bysshe Shelley, può usare la lingua straniera per diversi tipi di testo (e probabilmente proprio per questo motivo l'uso dell'italiano da parte di Shelley non ha un grande valore linguistico o letterario); e forse generalizzando un poco i risultati dell'indagine di Laura Colombo, si può affermare che nell'Ottocento francese non si ricorre all'italiano per fare letteratura 'alta' - essendo disponibile per questo una lingua di grande tradizione letteraria - ma piuttosto per la scrittura giornalistica e pubblicistica.

Le indagini di tipo letterario sull'eteroglossia possono comprendere - come mostrano nel volume gli interventi di Furio Brugnolo e di Francesco Rognoni - quel caso particolare della scrittura in lingua straniera che è l'autotraduzione. Nel rapporto fra testo originale e testo tradotto, la cultura occidentale ha solitamente e tradizionalmente visto nel testo di partenza la sede precipua della letterarietà e ha assegnato al testo di arrivo la funzione 'servile' di trasmettere i contenuti, fin dove è possibile, dell'originale e, con limitazioni ancora maggiori, riprodurne la forma. Le più moderne riflessioni sulla traduzione letteraria, invece, rivendicano un ruolo e un valore più rilevante al testo tradotto e, indi- 
rettamente, al traduttore come mediatore culturale; ${ }^{8}$ ciò vale nel caso particolare delle cosiddette 'traduzioni d'autore', ad esempio quando Eugenio Montale traduce T.S. Eliot, dove la traduzione può diventare riscrittura, ${ }^{9}$ ma non solo. Diverso è, tuttavia, analizzare criticamente l'autotraduzione, dove l'autore si ritrova a riscrivere se stesso: non è facile in tal caso determinare come si gioca il rapporto fra testo originale e testo tradotto, perché la differenza non sembra stare solo nella presunta, inferiore padronanza della lingua straniera da parte dello scrittore, la questione non sembra essere così banale, e ciò per diversi motivi. Lo scrittore che traduce sé stesso, infatti, può introdurre nel testo dei cambiamenti, operare degli scarti rispetto all'originale che un normale traduttore si vieterebbe di fare. E ciò anche perché, ovviamente, chi si autotraduce ha la massima consapevolezza dell'intento alla base del testo originale e del contesto culturale e letterario che lo ha prodotto. Inoltre, la scelta della lingua altra può essere dettata dal desiderio di dire qualcosa che nella lingua madre è indicibile, non si può o non si vuole dire. In ultima sintesi, e in modo ancora più profondo e radicale, va capito se la scelta dell'autotraduzione abbia un fondamento estetico e letterario o piuttosto psicologico e identitario. ${ }^{10}$

8 Dall'amplissima bibliografia disponibile su queste tematiche si possono selezionare due titoli rappresentativi: Maria Tymoczko, Enlarging translation, empowering translators, Manchester, St. Jerome Publishing, 2007, e Brian James Baer, "De-sacralizing the origin(al) and the transnational future of translation studies", Perspectives, 25 (2), 2017, pp. 227-244.

9 Si vedano: Rachel Meoli Toulmin, "Shakespeare ed Eliot nelle versioni di Eugenio Montale”, Belfagor, 26 (4), 1971, pp. 453-471; AA.VV., Eugenio Montale. Traduttore e tradotto, La Spezia, Edizioni Cinque Terre, 2003; Marco Sonzogni, "Debiti e doni della traduzione poetica: Montale tra T.S. Eliot e Samuel Beckett. Appunti su Montale traduttore e tradotto", The Italianist, 25 (2), 2005, pp. 173-207; Ernesto Livoni, "Montale traduttore di Eliot: una questione di 'belief'”, in Giuseppe Galigani (a cura di), Italomania(s): Italy and the English-speaking world from Chaucer to Seamus Heaney, Firenze, Mauro Pagliai Editore, 2007, pp. 139-160.

10 Il processo e i prodotti dell'autotraduzione sono diventati significativi oggetti di ricerca negli ultimi decenni. Tra i molti studi si possono citare: Rainier Grutman, "Auto-translation”, in Mona Baker e Kirsten Malmkjer (a cura di), Routledge encyclopaedia of translation studies, London/New York, Routledge, 1998, pp. 17-20; Mary Besemeres, Translating one's self: Language and selfbood in cross-cultural autobiography, Oxford/Bern, Peter Lang, 2002; Isabelle De Courtivron (a cura di), Lives in translation. Bilingual writers on identity and creativity, New York, Palgrave Macmillan, 2003; Jan Walsh Hokenson e Marcella Munson, The bilingual text. History and theory 
4. Volendo infine illustrare sinteticamente forme e modi della ricerca di taglio storico-culturale sull'eteroglossia, si deve fare riferimento agli studi e agli interessi che non si focalizzano sul tessuto linguistico o sulla natura propriamente letteraria del testo steso in lingua straniera, ma piuttosto sul contesto in cui l'eteroglossia ha trovato attuazione.

Per contesto, si può innanzitutto intendere, e si deve considerare, il prestigio della lingua straniera usata: tale prestigio può creare una situazione di egemonia di una lingua rispetto ad altre; un'egemonia che non è necessariamente culturale e letteraria, ma può anche essere sociale, politica ed economica, e ciò non di meno condizionare la decisione di usare la lingua straniera.

L'elemento del contesto è pure determinante quando la scelta dell'eteroglossia intende realizzare l'intento dello scrittore o scrivente straniero di calarsi in un altro mondo, in una cultura altra, per appropriarsene, per immedesimarsi con essa. Per fare un esempio, Gabriella Catalano ha sottolineato questo desiderio di integrazione da parte di Johann Joachim Winckelmann nei confronti del mondo italiano, e romano in particolare; un desiderio certo non sorprendente in un cultore della classicità letteraria e soprattutto artistica. Questo atteggiamento potrebbe anche spiegare, almeno in parte, l'uso della lingua ita-

of literary self-translation, Manchester, St. Jerome Publishing, 2007 (nuova edizione London/New York, Routledge, 2014); Alessandra Ferraro (a cura di), "L'autotraduzione nelle letterature migranti", numero speciale di Oltreoceano, 5, 2011; Simona Anselmi, On self-translation. An exploration in self-translators' teloi and strategies, Milano, LED, 2012; Anthony Cordingley (a cura di), Self-translation: Brokering originality in bybrid culture, Londra, Continuum, 2013; Michael Boyden e Liesbeth De Bleeker (a cura di), "Self-translation", numero monografico di Orbis Litterarum, 68 (3), 2013; Christian Lagarde e Helena Tanqueiro (a cura di), L'autotraduction: aux frontiers de la langue et de la culture, Limoges, Lambert-Lucas, 2013; Andrea Ceccherelli, Gabriella Imposti e Monica Perotto (a cura di), Autotraduzione e riscrittura, Bologna, Bononia University Press, 2013; Rainier Grutman e Trish van Bolderen, "Self-translation", in Sandra Bermann e Catherine Porter (a cura di), A companion to translation studies, Hoboken, NJ, Wiley Blackwell, 2014, pp. 323-332; Alessandra Ferraro e Rainier Grutman (a cura di), L'autotraduction littéraire. Perspectives théoriques, Parigi, Classiques Garnier, 2016; Giorgia Falceri, Eva Gentes e Elizabete Manterola (a cura di), "Narrazioni del sé e autotraduzioni", numero monografico di Ticontre. Teoria Testo Traduzione, 7, 2017; Gabriella Cartago e Jacopo Ferrari (a cura di), Momenti di storia dell'autotraduzione, Milano, LED, 2018; Fabio Regattin e Alessandra Ferraro (a cura di), Gli scrittori si traducono. Riflessioni, discorsi e conversazioni sull'autotraduzione da parte di chi la pratica, Città di Castello, Emil di Odoya, 2019. 
liana da parte di Lord Byron, transfuga dal mondo inglese. ${ }^{11}$ D'altra parte, però, il viaggiare a lungo o il vivere in Italia non porta necessariamente con sé questa integrazione; né è prova concreta P.B. Shelley che, nonostante gli interessanti esperimenti di eteroglossia a base italiana di cui parla Francesco Rognoni, manifesta esplicitamente il contrasto fra la sua adesione ammirata alle bellezze artistiche e naturali d'Italia e il suo distacco, che giunge ad essere una vera e propria avversione, nei confronti degli italiani e del contesto sociale in cui il poeta inglese si trova a vivere. ${ }^{12}$

Il caso paradigmatico di Shelley, che trascorse gli ultimi quattro anni della sua breve vita in Italia, dimostra come l'impiego della lingua straniera nella scrittura sia per lui indice di un legame, cercato e trovato, con una tradizione letteraria, e non con un contesto sociale. Molto dipende - pare necessario sottolineare - dalla vicenda biografica di chi viene a padroneggiare la lingua straniera ed è esposto al relativo contesto sociale: non si possono certo considerare allo stesso modo la conoscenza della lingua e la produzione poetica in italiano di John Milton $^{13}$ - vero poliglotta, che conosceva latino, greco, ebraico, francese, spagnolo, italiano e olandese, e che viaggia in Francia e Italia tra il

11 Sull'italiano di Lord Byron si veda Gabriella Cartago, "Le lettere dall'Italia e le lettere in italiano di Byron", in Furio Brugnolo e Vincenzo Orioles (a cura di), Eteroglossia e plurilinguismo letterario, vol. 1: L'italiano in Europa, Roma, Il Calamo, 2002, pp. 219-231.

12 Tale contrasto viene definito dagli studiosi della storia dei rapporti anglo-italiani mediante l'espressione "two Italies". Questa rimanda a una lettera che lo stesso Shelley scrisse nel dicembre 1818 all'amico Leigh Hunt, e che merita di essere qui citata almeno in parte perché vi viene menzionato anche Byron, 'corrotto' dal vivere in Italia fra gli italiani: "There are two Italies; one composed of the green earth \& transparent sea and the mighty ruins of antient times, and aerial mountains, \& the warm \& radiant atmosphere which is interfused through all things. The other consists of the Italians of the present day, their works \& ways. The one is the most sublime \& lovely contemplation that can be conceived by the imagination of man; the other the most degraded disgusting \& odious. - What do you think? young women of rank actually eat - you will never guess what - garlick. Our poor friend Lord Byron is quite corrupted by living among these people; $\&$ in fact is going on in a way not very worthy of him." (F. L. Jones, a cura di, The letters of Percy Bysshe Shelley, Oxford, Clarendon Press, 1964, vol. 2, p. 67).

${ }^{13}$ Com'è noto, la produzione poetica di John Milton comprende - oltre a due componimenti in inglese ma dal titolo italiano, rispettivamente L'Allegro e Il Penseroso [sic] - cinque sonetti e una canzone in lingua italiana, pubblicati per la prima volta nell'edizione dei Poems of Mr. John Milton, Londra, Humphrey Moseley, 1645, pp. 45-48. 
1638 e il 1639 - e quella di Manuel Lassala che, come ci spiega Maurizio Fabbri, trascorre esule in Italia gran parte della propria vita. Detto altrimenti, e in termini più generali, si tratta di capire se la scelta dell'eteroglossia letteraria dipenda da un intento programmatico dell'autore o dalle vicende pratiche della sua vita.

Anche considerando le esperienze dei viaggiatori del Grand Tour e le documentazioni testuali che spesso essi ci hanno lasciato, si riscontra un variabile e differenziato desiderio di inserirsi in una realtà altra e di riflettere tale coinvolgimento nella pratica dell'eteroglossia, anche come modo di dar prova dell'arricchimento culturale conseguente all'esperienza del viaggio all'estero. Il viaggiatore spesso studiava l'italiano durante il proprio soggiorno, per cui poteva avere un senso stendere il resoconto di viaggio nella lingua straniera. Senza arrivare a questo, i diari e le lettere di molti viaggiatori, seppur scritti nella lingua madre, potevano raccogliere parole, espressioni, o brevi frasi in italiano, spie del contatto interlinguistico; ${ }^{14}$ ma abbiamo esempi di viaggiatori stranieri refrattari ad avvicinarsi alla lingua italiana: uno è lo storico e politico Edward Gibbon (1737-94), il famoso autore della History of the Decline and Fall of the Roman Empire, che in Italia usa per comunicare il francese appreso durante un precedente lungo soggiorno in Svizzera; un altro è James Boswell (1740-1795), il futuro biografo di Samuel Johnson, che durante il Grand Tour in Italia si intende in latino coi propri interlocutori, anche se prende lezioni d'italiano.

5. Questa veloce e sintetica serie di riflessioni sul fenomeno, indubbiamente interessante, dell'eteroglossia letteraria può trovare il suo compimento in alcune considerazioni conclusive di carattere generale.

Va innanzitutto sottolineato che, sempre nell'analisi del testo letterario, ma forse ancora di più nel caso particolare del testo che nasce dalla scelta dell'eteroglossia, gli approcci di tipo linguistico, critico-letterario e storico-culturale devono necessariamente essere integrati fra loro per portare a una piena e convincente interpretazione del testo.

14 Questi materiali linguistici sono schedati e commentati in Gabriella Cartago, Ricordi d'italiano. Osservazioni intorno alla lingua e italianismi nelle relazioni di viaggio degli inglesi in Italia, Bassano del Grappa, Ghedina \& Tassotti Editori, 1990. 
Secondariamente, va ricordato che l'autore del testo in lingua straniera - sia questi un letterato, un uomo di cultura o semplicemente una persona istruita - è di necessità un poliglotta. Ovviamente deve essere bilingue e, oltre alla lingua madre, deve in qualche modo padroneggiare la lingua straniera impiegata nella stesura del testo; ma ancor più di questo si può affermare, seppure generalizzando, che chi si cimenta - soprattutto chi si è cimentato nel passato - in questa particolarissima esperienza di scrittura creativa deve aver avuto una formazione linguistica ampia, nutrita magari dalle lingue classiche accanto a una lingua moderna, o più d'una.

In terzo luogo, bisogna tenere in debito conto il momento storico-culturale in cui si attua la scelta dell'eteroglossia. Già si è detto come del Cinque-Seicento è ben noto il diffuso plurilinguismo, che rende facile la scelta dell'eteroglossia letteraria; il Sette-Ottocento vede tale scelta quantitativamente più limitata, ma forse proprio per questo particolarmente significativa e interessante; diversi scrittori del Novecento, secolo della crisi degli stati nazionali correlata al dramma dei due conflitti mondiali, scoprono o riscoprono nella babele delle lingue una possibile strada verso la difficile ricerca del senso delle cose e della vita. Tuttavia, la mescolanza delle lingue e la tensione fra le lingue si ritrovano anche nel passato più lontano come nel presente più vicino: infatti ci si può riferire, da una parte al passaggio dal medioevo alla prima età moderna con l'affermazione delle lingue nazionali rispetto sia alle varietà locali sia all'uso del latino come lingua sovranazionale, e dall'altra alla tensione cui sono sottoposte oggi le lingue europee - anche le lingue nazionali di grande tradizione e cultura - in un certo qual modo condizionate dalle lingue radicalmente altre che vengono dal sud e dall'est del mondo ${ }^{15}$ e dal dilagare incontrollato dell'inglese come lingua sovranazionale. In questo variegato contesto sociolinguistico e interculturale, riflettere sull'eteroglossia non sembra essere, non è, un puro esercizio di ricostruzione storico-culturale ma può anche illuminare qualche aspetto della contemporaneità.

15 L'esperienza della scrittura eteroglossa a base italiana non è un fatto solo del passato e dello spazio culturale europeo, come ci mostra il progetto BASILI\&LIMM (Banca Dati degli Scrittori Immigrati in Lingua Italiana e della Letteratura Italiana della Migrazione Mondiale - http://basili-limm.el-ghibli.it), avviato da Armando Gnisci nel 1997, che attualmente censisce ben 570 scrittori di circa 50 diverse lingue madri. 
Come considerazione ultima di questo contributo è probabilmente opportuno accennare verso quali ambiti e questioni la ricerca sull'eteroglossia letteraria potrebbe essere ulteriormente indirizzata. Si potrebbe tentare di determinare, ad esempio, se la scelta dell'eteroglossia si sostanzia naturalmente verso forme letterarie 'alte' o è altrettanto praticata per forme più basse e popolari; se esistono delle lingue che più facilmente si prestano a essere usate per l'eteroglossia con finalità letteraria o paraletteraria, e perché; se la scrittura in lingua straniera è più facile per gli uomini o per le donne, oppure questa variabile non è significativa; se l'opzione per l'eteroglossia può essere correlata a particolari situazioni o vicende sociali e politiche; se, al di là di quanto già sappiamo in relazione al mondo europeo occidentale, esiste un tempo o uno spazio storico-culturale più favorevole a questo tipo di esperienza letteraria, e perché. Forse si potrebbe dare una risposta a queste e altre domande se si arrivasse alla compilazione di un repertorio, ragionevolmente esaustivo, di testi dell'eteroglossia letteraria. Sarebbe già sarebbe un ottimo risultato raccogliere questi testi con riferimento a un'unica lingua straniera utilizzata, ad esempio l'italiano; anche se confronti fra produzioni diverse - il testo italiano di un inglese e il testo francese di un tedesco - potrebbero farci meglio capire quale sia l'essenza dell'eteroglossia letteraria; e una schedatura sistematica e completa potrebbe permettere di analizzare e rendere palesi delle tendenze con cui confrontare la singola attuazione dell'eteroglossia.

Al di là delle presenti e future acquisizioni della critica, pare opportuno concludere questa breve rassegna, e contemporaneamente il volume che raccoglie saggi sull'eteroglossia letteraria a base italiana, dando voce a una scrittrice straniera che recentemente ha fatto la scelta di scrivere in lingua italiana, e ha fatto di tale scelta l'oggetto di un suo libro. Mi riferisco a Jhumpa Lahiri, scrittrice di origine bengalese nata in Inghilterra e cresciuta negli Stati Uniti; dopo una raccolta di racconti brevi e tre romanzi in lingua inglese acclamati dalla critica, la scrittrice si appassiona alla lingua italiana, e si trasferisce a Roma per impadronirsene al meglio. Il risultato è il volume In altre parole, scritto in italiano e pubblicato in Italia nel 2015, per poi comparire l'anno successivo in un'edizione con traduzione a fronte non sua. In altre parole è la riflessione dell'autrice sulle motivazioni profonde e sugli esiti letterari circa la propria scelta dell'eteroglossia, come mostra il seguente brano tratto dal capitolo intitolato "Il riparo fragile": 
Quando scrivo in italiano mi sento un'intrusa, un'impostora. Sembra un compito contraffatto, innaturale. Mi accorgo di aver oltrepassato un confine, di sentirmi persa, di essere in fuga. Di essere completamente straniera.

Quando rinuncio all'inglese rinuncio alla mia autorevolezza. Sono traballante anziché sicura. Sono debole.

Da dove viene l'impulso di allontanarmi dalla mia lingua dominante, la lingua da cui dipendo, da cui provengo come scrittrice, per darmi all'italiano?

Prima di diventare un'autrice mi mancava un'identità chiara, nitida. E' stato attraverso la scrittura che sono riuscita a sentirmi realizzata. Ma quando scrivo in italiano non mi sento così.

Cosa vuol dire scrivere senza la propria autorevolezza? Posso definirmi un'autrice, senza sentirmi autorevole?

Com'è possibile, quando scrivo in italiano, che mi senta sia più libera sia inchiodata, costretta?

Forse perché in italiano ho la libertà di essere imperfetta.

Come mai mi attrae questa nuova voce, imperfetta, scarna? Come mai mi soddisfa la penuria? Cosa vuol dire rinunciare a un palazzo per abitare quasi per strada, sotto un riparo così fragile?

Forse perché dal punto di vista creativo non c'è nulla di tanto pericolo quanto la sicurezza.

Mi chiedo quale sia il rapporto tra libertà e limitazioni. Mi chiedo come una prigione possa somigliare al paradiso. ${ }^{16}$

16 Jhumpa Lahiri, In Other Words, London, Bloomsbury, 2016, pp. 81, 83. Questa edizione con testo a fronte italiano-inglese e traduzione inglese di Ann Glodstein segue la prima edizione italiana di In altre parole, Milano, Guanda, 2015. 